\title{
Limits on the dark matter content of globular clusters
}

\author{
H. Baumgardt, ${ }^{1}$ P. Côté, M. Hilker, M. Rejkuba, S. Mieske, \\ S. G. Djorgovski and P. Stetson \\ ${ }^{1}$ Argelander-Institut für Astronomie, Universität Bonn, Auf dem Hügel 71, 53121 Bonn, \\ Germany
}

\begin{abstract}
We have measured the velocity dispersion of the Galactic globular cluster NGC 2419 to determine if a substantial amount of dark matter is present in this cluster. NGC 2419 is one of the best globular clusters to look for dark matter due to its large mass, long relaxation time and large Galactocentric distance, which makes tidal stripping of dark matter unlikely. Our results can be summarized as follows. (i) We found a global velocity dispersion of $4.14 \pm 0.48 \mathrm{~km} \mathrm{~s}^{-1}$, which leads to a total cluster mass of $(9.02 \pm 2.22) \times 10^{5} \mathrm{M}_{\odot}$ and implies a global mass-to-light ratio of $2.05 \pm 0.50 \mathrm{M}_{\odot} / \mathrm{L}_{\odot}$. (ii) Our derived mass-to-light ratio is completely consistent with the mass-to-light ratio of a standard stellar population at the metallicity and age of NGC 2419. In addition, the mass-to-light ratio of NGC 2419 does not increase towards the outer cluster parts. (iii) We can therefore rule out the presence of a dark-matter halo with a central density greater than about $0.02 \mathrm{M}_{\odot} \mathrm{pc}^{-3}$. Similar limits are found for other halo globular clusters, like Pal 14 . These observations therefore indicate that NGC 2419 and other halo globular clusters did not form at the centers of dark-matter halos similar to those surrounding dwarf galaxies. Instead, an origin driven by gas-dynamical processes during mergers between galaxies or proto-galactic fragments seems to be the more likely explanation for the formation of even the lowest-metallicity globular clusters.
\end{abstract}

Keywords. globular clusters: individual (NGC 2419), dark matter

The full poster (in pdf format) is available at http://www . astro.iag. usp.br/ iaus266/Posters/pBaumgardt.pdf. 